\title{
LA COOPERACIÓN CIENTÍFICA Y TECNOLÓGICA DE MÉXICO, CANADÁ Y ESTADOS UNIDOS EN LA ERA TRUMP. ¿RETOS NUEVOS, O QUÉ TAN NUEVOS? ${ }^{1}$
}

\author{
Scientific and technological cooperation between Mexico, \\ Canada and the United States in the Trump era. \\ New challenges or not so new?
}

Juan Carlos Moreno-Brid², Pedro Enrique Armendares ${ }^{3}$, Isabel Salat ${ }^{4}$

Fecha de recepción: 14 de noviembre del 2017

Fecha de aceptación: 10 de abril del 2018

1- Los autores quieren agradecer a la Dra. Gabriela Dutrénit su generosa orientación, a través de diversas sesiones, en el importante tema de los sistemas nacionales de innovación en México y América Latina.

2- Nacionalidad: Mexicana. Grado: Doctorado, Especialización: Economía. Adscripción: Universidad Nacional Autónoma de México. Correo electrónico: juancarlosmorenobrid@gmail.com. (D) ORCID: http://orcid.org/0000-0003-2238-0019

3- Nacionalidad: Mexicana. Grado: Maestría. Especialización: Comunicación y Ciencias Sociales. Adscripción: Investigador independiente. Correo electrónico: nanuc@mac.com. (iD ORCID: http://orcid.org/0000-0003-2952-0416

4- Nacionalidad: Mexicana. Grado: Licenciatura. Especialización: Economía. Adscripción: Facultad de Economía, UNAM. Correo electrónico: isabelsalat@gmail.com. (D) ORCID: https://orcid.org/0000-0002-6035-7891 


\section{Introducción}

$\mathrm{M}$

ucho se ha escrito sobre la integración de América del Norte y su potencial para el crecimiento económico y el bienestar social de los tres países de la región, sobre todo después de la implementación del Tratado de Libre Comercio (TLCAN) hace más de 20 años. Sin embargo, la literatura ha prestado menos atención a un aspecto que resulta clave para el progreso y desarrollo de la región: la cooperación subregional en el campo de la ciencia, la tecnología y la innovación (CTI).

Pese a que dicha cooperación no fue un tema que se considerara crucial en la negociación de 1993, los entonces impulsores del TLCAN y de las reformas de mercado auguraban que el Tratado alentaría la innovación y el comercio de bienes y servicios protegidos por los derechos de propiedad intelectual (véase TLCAN, 1993).

El TLCAN y las reformas de mercado permitieron consolidar el cambio en el modelo de especialización productiva de la economía mexicana, desde una concentración en las exportaciones no petroleras hasta convertirse en una potencia exportadora de manufacturas. México es hoy uno de los países que más exportan de América Latina; sin embargo, este dinamismo exportador ha estado lejos de asegurar un crecimiento suficiente para satisfacer las necesidades del país. Aunado a ello, ese crecimiento ha sido acompañado por una creciente concentración del ingreso. Así, los niveles de pobreza permanecen en niveles cercanos a los de 1992.

En términos de desarrollo, el desempeño de México vis a vis Estados Unidos y Canadá dista de ser satisfactorio. Cuando se implementó el Tratado, se pensaba que contribuiría a generar el desarrollo armónico entre México, Canadá y Estados Unidos (TLCAN, 1993). Hoy en día, las brechas se han acentuado. Si bien la cooperación en CTI no fue un objetivo en sí mismo, se pensaba que la economía mexicana avanzaría en términos de desarrollo tecnológico. Sin embargo, México sigue muy lejos de sus socios comerciales en términos de innovación.

Este trabajo parte de ese escenario para explorar el margen para ampliar la cooperación trilateral, y considera al TLCAN como un elemento complementario pero no la base de la competitividad de la economía mexicana.

En la primera sección, discutimos la importancia de la cooperación en la CTI para el desarrollo económico y la competitividad, en el marco de la economía mexicana, y reconociendo que esa cooperación está determinada por diversos agentes económicos, tanto privados como públicos, que interactúan en el Sistema Nacional de Innovación (SNI).

En la segunda sección exponemos los antecedentes de la cooperación con América del Norte, abordamos las asimetrías en CTI que existen entre los tres países miembros del TLCAN, y exploramos espacios de oportunidad para avanzar en el desarrollo de esa cooperación.

Finalmente, el texto analiza dos componentes que podrían obstaculizar esas oportunidades. Uno está ligado a fallas estructurales que han sumido a la economía mexicana en una senda de muy bajo crecimiento, pobreza y elevada desigualdad. El otro, de tipo coyuntural, surge del giro radical que ha dado el gobierno de Trump a la política estadounidense sobre el TLCAN, incluyendo comercio e inversión subregional, y de hecho la relación con México en general. 


\section{E1 sistema nacional de innovación y la cooperación en CTI}

La literatura aborda la cooperación en CTI desde distintas perspectivas. ${ }^{56}$ En nuestro marco de análisis rescatamos el impacto del cambio tecnológico y la transferencia de tecnología entre países a través de los acuerdos de libre comercio (ALC). Los ALC pueden dinamizar la adopción de nuevas tecnologías a través del comercio de bienes y servicios de alta tecnología e inversión extranjera directa (IED), entre otros factores. Si bien es cierto que los ALC pueden jugar un papel importante para facilitar la adopción de nuevas tecnologías entre países, los procesos de innovación dependen de múltiples factores; entre ellos, la capacidad de los países menos desarrollados de apropiarse de dichos conocimientos.

Los procesos de innovación son fenómenos complejos que implican la interacción de múltiples agentes económicos, tanto públicos y privados, en un sistema nacional de innovación (SNI) (Freeman 1987; Lundvall 1992; Nelson 1993, y Metcalfe 1995). En este contexto, el SNI es el conjunto de agentes, instituciones, articulaciones y prácticas sociales vinculados a la actividad innovadora al interior de los países (Dutrenit 1994).

Nuestro enfoque vincula el SIN con la cooperación en CTI para la manera en la que las acciones de distintos agentes económicos inciden sobre el progreso tecnológico y la innovación, como resultado de una compleja interacción entre actores sociales (OECD, 1997) tales como empresas, instituciones de enseñanza superior y centros de investigación.

Los procesos de innovación y la adopción de nuevas tecnologías son fundamentales para elevar la competitividad y el crecimiento en economías globalizadas con modelos de especialización productiva liderada por las exportaciones, como la mexicana.

\section{Cooperación en CTI entre México, Estados Unidos y Canadá}

La cooperación científica institucional con Estados Unidos se inició un par de años después de la creación del CONACYT, con el Acuerdo de Cooperación Científica y Técnica en 1972. Este acuerdo lanzó el Programa de cooperación correspondiente con el objetivo de incrementar las capacidades científicas y tecnológicas de ambos países y aprovechar mejor "el intercambio de personas, ideas, habilidades experiencias e información" (Antal y Aroche, 2010; SRE, 1972). El Programa promovió proyectos específicos entre universidades e instituciones de investigación de ambos países. Uno de los logros notables de esta vinculación es el Gran Telescopio Milimétrico (GTM) Alfonso Serrano, construido en la Sierra Negra de Puebla como proyecto conjunto de la Universidad de Massachusetts y el Instituto Nacional de Astrofísica, Óptica y Electrónica (INAOE).?

5- Por ejemplo, desde las redes de cooperación Sebastián (2000); desde la perspectiva de la asociación estratégica entre empresas Martínez-Noya y García-Canal (2017); y para un análisis sobre el impacto de los Programas Nacionales y Regionales de Innovación sobre la Cooperación Internacional en Innovación Fernández-Ribas y Shapira (2009).

6- Para una perspectiva histórica de CTI en América Latina, ver Lemarchand (2010).

Para una revisión de la literatura sobre cooperación internacional en ciencia y tecnología ver Oregioni y López (2013).

7- Ver, Large Millimeter Telescope . 2017. Gran Telescopio Milimétrico Alfonso Serrano. http://www.lmtgtm.org/ (26 de agosto, 2017). 
A partir de las reformas de mercado a mediados de los 1980s, profundizadas por las administraciones subsiguientes, incluyendo de manera destacada el TLCAN en 1994, se produjo una restructuración profunda de la economía mexicana. La apertura tanto comercial como financiera, junto con la reorientación hacia el mercado externo, la integró aún más a la economía de Estados Unidos. Las exportaciones, las importaciones y los flujos de inversión extranjera de México se concentraron mayoritariamente hacia ese país. Y, si bien la ciencia y la tecnología no fueron prioridades del TLCAN, el acercamiento entre ambas economías potenció los flujos e intercambio de conocimientos y el aprovechamiento conjunto de innovaciones. Ello ocurrió con más intensidad en campos o sectores considerados estratégicos como medio ambiente, energía y telecomunicaciones, mismos que ya eran así señalados en el Acuerdo México-Estados Unidos de 1972 (Dutrenit y Suárez, 2014).

Diez años después de la entrada en vigor del TLCAN, en 2004, México firmó una Alianza con Canadá para impulsar la cooperación bilateral entre gobiernos, sector privado y otros actores, a través de instituciones de educación superior e investigación. La Alianza stableció ocho grupos de trabajo temáticos, incluyendo desarrollo, energía, medio ambiente, recursos naturales, transporte y salud, entre otros. ${ }^{8}$

En 2005 los tres países del TLCAN reforzaron los compromisos establecidos por el Tratado con la firma de la Alianza para la Seguridad y Prosperidad de América del Norte (ASPAN). Conocida en su momento como TLCAN Plus, surgió como iniciativa de los entonces Presidentes Vicente Fox y George Bush y el Primer Ministro George Martin. Además de la seguridad, se buscó fortalecer la cooperación para desarrollar el capital humano en educación superior, ciencia y tecnología, y de manera específica en temas como biotecnología, energía, transporte, medio ambiente y salud, entre otros (US State Department, 2005).

El intercambio en CTI con Canadá dio otro paso en 2011, cuando se firmó el Acuerdo de Cooperación en Materia de Investigación e Innovación entre el Gobierno de Quebec y México. ${ }^{9}$ Su objetivo es desarrollar actividades y proyectos conjuntos que contribuyan a reforzar la investigación científica y tecnológica. Las áreas de interés común para esta cooperación son biotecnología y agronomía; biología y química; ciencias de la tierra; energía; medio ambiente; medicina y ciencias de la salud; ciencias físicas y matemáticas; nuevos materiales; nanotecnologías; tecnologías de información y telecomunicaciones; ingeniería y tecnologías industriales, y sector aeroespacial.

El mecanismo de cooperación bilateral en CTI más importante hasta ahora es el Foro Bilateral México-Estados Unidos sobre Educación Superior, Innovación e Investigación (FOBESII), establecido en 2013. Tiene el objetivo de promover capital humano y el desarrollo de ambos países, así como de contribuir a transformar a América del Norte en una región del conocimiento (FCCyT, 2013). En particular, es consistente con el programa mexicano Proyecta 100,000, cuya meta es que para 2018 ese número de mexicanos haya sido enviado a estudiar a Estados Unidos y 50,000 estadounidenses hayan ido a estudiar a México.

Tan solo en 2014 ambos gobiernos, en colaboración con sus respectivos sectores académico y privado, propiciaron estancias de intercambio en Estados Unidos para más de 27,000 estudiantes y profesores. ${ }^{10}$

8- Ver, SRE. 2016. Alianza México-Canadá. https://mex-can.sre.gob.mx/index.php/alianza-mexico-canada (23 de agosto, 2017).

9- Ver, CONACYT. 2011. Estrechan vínculos de cooperación México-Quebec. http://2006-2012.conacyt.gob.mx/comunicacion/comunicados/Paginas/35-11.aspx (27 de agosto, 2017).

10- Ver, INROADS. 2013. El foro bilateral México-Estados Unidos sobre educación superior, innovación e investigación: conectamos hoy a los líderes del mañana. https://www.inroads.org.mx/archivos/FOBESII-JoinStatement.pdf (12 de agosto, 2017). 
Entre los numerosos resultados concretos de alta resonancia destaca el trabajo del equipo multidisciplinario de la Universidad de California (campus Riverside y San Diego), el Centro de Investigación Científica y de Educación Superior de Ensenada (CICESE), la UNAM, y el Instituto Nacional de Astrofísica, Óptica y Electrónica (INAOE). Este equipo ha desarrollado un implante de cráneo, fabricado con cerámica transparente, que permitirá dar tratamiento no invasivo mediante laser a pacientes con padecimientos neurológicos que ponen en riesgo la vida, tales como cáncer cerebral y embolia. El proyecto, llamado Ventana al cerebro, está auspiciado por la Fundación Nacional para la Ciencia de Estados Unidos y por el CONACYT. ${ }^{11}$

Mientras tanto, diversas universidades e instituciones académicas de Estados Unidos están estableciendo campus, centros de investigación conjunta u oficinas en México. Por ejemplo, la Universidad de Arkansas tiene un campus en Querétaro, y la Universidad Estatal de Colorado estableció una extensión internacional en Todos Santos, Baja California Sur, dedicada a la enseñanza y a la investigación.

La cooperación de México con sus socios del TLCAN se ha realizado en gran medida a través de sus instituciones públicas y del Sistema Nacional de Investigadores (SNI). El SNI se originó en el Consejo Nacional de Ciencia y Tecnología (CONACYT), el cual se fundó en 1970 como primer paso para impulsar la Ciencia y Tecnología (CyT) como parte de las políticas públicas. El CONACYT tiene el objetivo de elevar el nivel de vida de la población impulsado a través del aumento de la capacidad científica y tecnológica del país, ${ }^{12}$ y es la institución mexicana más importante la elaboración y promoción de políticas vinculadas a CyT.

El Foro Consultivo Científico y Tecnológico (FCCT) es otra iniciativa relevante para la implementación de políticas públicas sobre conocimiento científico, desarrollo tecnológico innovación como motores del crecimiento económico. El FCCT está alineado con el Plan Nacional de Desarrollo y atiende las metas en materia de CTI del Poder Ejecutivo, el CONACYT y el Consejo General de Investigación Científica, Desarrollo Tecnológico e Innovación. Funciona como puente entre actores públicos y privados incluyendo, entre los primeros, el Instituto Politécnico Nacional (IPN), la Universidad Nacional Autónoma de México (UNAM) y la Academia nacional de Ciencias, y entre los segundos la Cámara Nacional de la Industria de la Transformación (CANACINTRA), la Confederación de Cámaras Industriales (CONCAMIN) y la Confederación Patronal de la República Mexicana (COPARMEX), entre otros.

\section{Retos estructurales y coyunturales: la economía mexicana y el factor Trump}

A raíz del TLCAN y las reformas económicas para reducir la participación del Estado en la economía, se esperaba que la mayor parte de la inversión en ciencia y tecnología fuera realizada por el sector privado. Asimismo, se esperaba que el aumento en la IED permitiría la modernización de la estructura productiva.

11- Ver, Univesity of California. 2016. Scientists move closer to developing therapeutic window to the brain. https://ucrtoday.ucr.edu/38537 (15 de agosto, 2017).

12- Ver: CONACYT. 2017. Consejo Nacional de Ciencia y Tecnología. https://2001-2009.state.gov/p/wha/rls/prsrl/2005/69850.htm (25 de agosto, 2017). 
Así, el TLCAN contribuiría a la cooperación regional en CTI, al crecimiento económico y la aumento de la competitividad, con énfasis en los sectores exportadores. Bajo esa perspectiva, la cooperación en CTI medida a través de la transferencia tecnológica parece haber sido relativamente exitosa, aunque en distintos grados para cada país del TLCAN (Park, 2011).

México es receptor, pero no creador, de grandes flujos de tecnología. En Canadá, los flujos de tecnología están asociados al TLCAN y a reformas a la propiedad intelectual. Para Estados Unidos, en cambio, el nivel de comercio de tecnología con los miembros del TLCAN no es muy distinto que con el resto del mundo (en particular con Japón y Europa).

Park (2011) encuentra muestran que, en general, el comercio en tecnología aumentó considerablemente después de la entrada en vigor del Tratado. La mayoría de las ganancias corresponden a México, ya que el comercio en tecnología entre Canadá y Estados Unidos era importante aún antes del TLCAN. Este análisis es consistente con la evolución de las exportaciones de alta tecnología como proporción de las exportaciones manufactureras en México posterior al Tratado (Park 2011).

El porcentaje de exportaciones de alta tecnología aumentó de manera constante a partir de 1991 (menos de 10\%) hasta 1995 (15\%) y, ya con el TLCAN en vigor, hasta un pico de cerca de $22 \%$ en 2000. Desde entonces ha descendido al 15\%, en 2016.

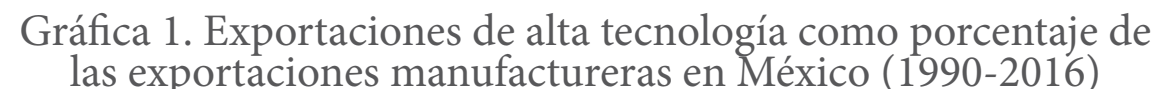
las exportaciones manufactureras en México (1990-2016)

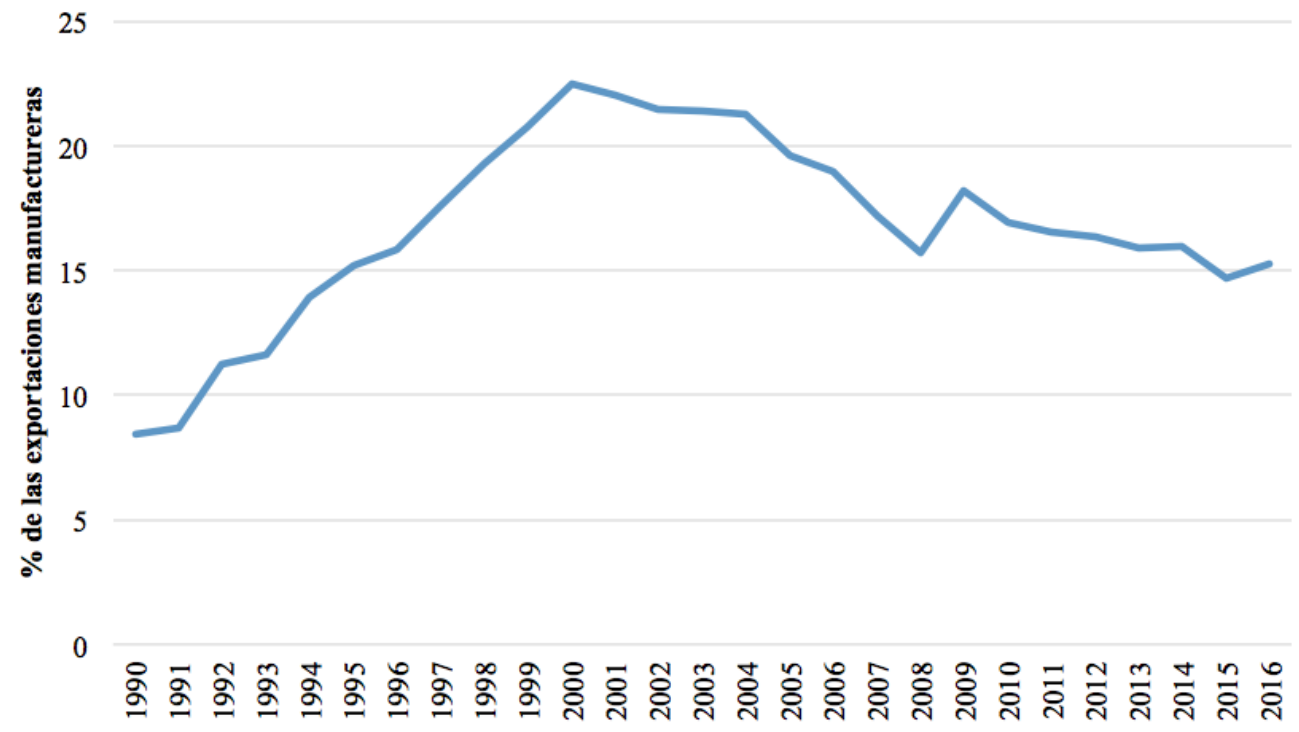

Fuente: Elaboración propia con datos de World Bank Statistics.

En contraste con las grandes expectativas de algunos actores sobre el potencial del TLCAN y las reformas de mercado para contribuir al crecimiento y la productividad de la economía mexicana, persisten grandes brechas entre los tres países de América del Norte. Esto sugiere que la liberalización comercial ha contribuido poco a mejorar el nivel de desarrollo México (véase gráfica 2). 
Gráfica 2. PIB per cápita y productividad laboral de México frente a Estados Unidos 1991-2016 (Dólares a precios constantes de 2010)

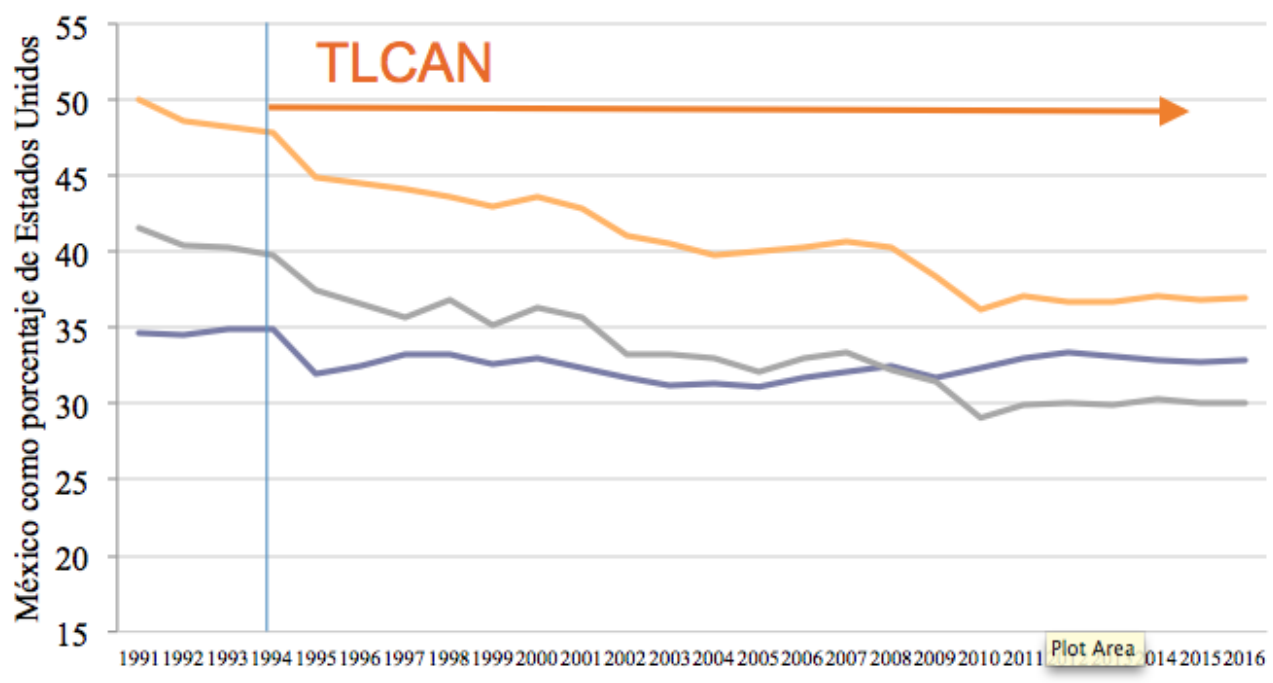

— PIB per cápita ——Producto por trabajador ——Producto por hora trabajada

Fuente: Elaboración propia con datos de OECD Statistics. Véase también Moreno-Brid, Blecker y Salat (2017).

Algunos autores argumentan que el magro desempeño de la productividad está relacionado con los bajos niveles de innovación, relacionados a su vez con factores institucionales que limitan el proceso de acumulación tecnológica (Dutrénit, 1994). El potencial de la cooperación en CTI depende de al menos dos factores. Uno, qué industrias absorben los avances tecnológicos y su vínculo con la generación de valor agregado y, el segundo, la inversión interna en investigación y desarrollo (IyD). En el primer caso, la estructura productiva se ha mantenido altamente dependiente de la importación de insumos. Además, pese a que los flujos de IED incrementaron la inversión física e incentivaron la incorporación de conocimiento así como el uso de nuevas tecnologías para la producción y comercialización de bienes y servicios, no ha desarrollado efectos sinérgicos sobre el conjunto de la actividad productiva, estando vinculados principalmente al comercio internacional o los servicios financieros (Dutrénit, Capdevielle, Corona et al., 2010). Por tanto, se requiere también que el desarrollo científico interactúe con otros sectores para permitir encadenamientos productivos que impulsen la generación de valor agregado (Cimoli, 2000).

El rezago de México en CTI se refleja en el número de artículos publicados en revistas indexadas y el número de patentes obtenidas. Así, por ejemplo, en 2013 los investigadores de Estados Unidos publicaron en promedio 0.31 artículos en revistas indexadas (per cápita); los de Canadá 0.43, y los de México 0.03. Un porcentaje importante de los artículos publicados en revistas indexadas de México (15.7\% en 2013) se escriben en colaboración con investigadores de Estados Unidos. El porcentaje de tales artículos con investigadores de Canadá es mucho menor (2.6\% en 2013) (Dutrénit y Suárez, 2014). La colaboración trilateral en artículos producidos en México fue aún menor (1.2\%). Estos bajos índices de colaboración implican 
que existe una oportunidad para incrementar la investigación y publicación conjunta, para lo que se requieren programas o plataformas específicas para promover esas actividades (Dutrénit y Suárez, 2010). De manera similar, el promedio anual de patentes en Estados Unidos fue 0.0197 (siempre per capita), comparado con 0.043 en Canadá y 0.0002 en México.

Un factor clave para el rezago de México en CTI es que la inversión pública en IyD de México es muy baja, muy lejana a $1 \%$ de PIB que ordena la Constitución La inversión privada en este campo también está muy por debajo de la de casi todos los países de la OCDE y del grupo BRICS (OCDE, 2017). Más aún, en 2017 México estuvo en el lugar 58 del Índice Global de Innovación, muy por debajo de Estados Unidos (cuarto lugar) y Canadá (décimo octavo lugar).

Mientras tanto, a nivel interno, más de 40 por ciento de los investigadores de alta calidad trabajan en instituciones de la Ciudad de México. La concentración regional de este tipo de indicadores de innovación como debilitan la competitividad nacional y los esfuerzos de desarrollo (Gurría, 2009)

Otro lastre del sistema nacional de innovación de México es la baja sinergia entre sus actores, a diferencia de lo que ocurre en Estados Unidos y Canadá, donde hay relaciones más intensas y frecuentes entre universidades, laboratorios, empresas, gobierno, agencias de financiamientos y complejos industriales (Niosi, 2000; Mowery y Rosenberg, 1993; Atkinson, 2014). En México, las relaciones entre el sector privado y las universidades e instituciones de investigación públicas son endebles. Esto es preocupante, ya que es en estas instituciones donde se desarrolla principalmente la innovación.

Así, el desarrollo de la ciencia, tecnologías e innovación en México es obstaculizado por características estructurales de su economía, poca coordinación entre los actores relevantes, baja inversión pública y escasa participación del sector privado (se recomienda leer Sánchez y García, 2015).

En una óptica constructiva, estas mismas asimetrías pueden verse como áreas de oportunidad para promover los intercambios, movimientos y flujos de conocimiento entre los tres países. Tema crucial es identificar los incentivos y marcos institucionales que los podrían potenciar y concretar de manera efectiva en beneficio de la población de la región.

En este contexto, el FOBESII puede ser un instrumento clave para el desarrollo económico y el bienestar social sostenido de la región a través de la formación de capital humano, la investigación y la innovación (FCCyT, 2013). Una acción importante para la consolidación estratégica del Foro sería integrar plenamente a Canadá al Foro. Esta vertiente cobra mayor peso en la medida que la colaboración de los socios mexicanos con el sector gubernamental de Estados Unidos se complique debido a las políticas de Trump. En ese escenario, las oportunidades de colaboración en CTI de México y Canadá de los diferentes actores de sus sistemas nacionales de innovación -por no subrayar solamente a los sectores académicos- deberían explorarse pues podrían ser muy relevantes en el futuro cercano.

Desde mediados de los años ochenta, el crecimiento de la economía mexicana se apoya primordialmente en dos factores. Por una parte, se redujo la injerencia del Estado en la esfera productiva en un marco de apertura comercial y financiera. Por otra, se buscó aprovechar la posición geográfica del país y la abundancia de mano de obra barata para convertir a México en una plataforma de exportaciones a Estados Unidos que, se pensaba, arrastrarían al resto de la economía hacia una expansión elevada y sostenida. Como resultado, actualmente más de $80 \%$ las exportaciones mexicanas se dirigen a Estados Unidos (sobre el crecimiento en México ver Sánchez y Moreno-Brid, 2016). 
Por otra parte, el ritmo de actividad de la manufactura mexicana está condicionado por el dinamismo industrial de Estados Unidos. De igual forma, la mayor proporción de inversión extranjera directa a México proviene de ese país, así como la mayoría de las remesas familiares. Esas remesas representan cerca de 6\% de los ingresos de la cuenta corriente de México, y son recibidas en buena medida por habitantes de regiones pobres. Todas esas fuentes de divisas de la economía mexicana son sumamente vulnerables a la evolución de la economía estadounidense y a cambios en sus políticas de comercio exterior, de inversión extranjera directa y de migración. Y todas han sido puestas en entredicho por el discurso y las primeras medidas proteccionistas y antiinmigrantes de Trump.

En materia de la relación comercial, Estados Unidos convocó -es un decir- a México y a Canadá a renegociar el TLCAN y puso sobre la mesa los cambios que pretende hacer al Tratado. Varios de ellos implican grandes ventajas para Estados Unidos y seguramente son inaceptables para México y Canadá. ${ }^{13}$ Trump también ha sugerido que aun después la renegociación su país saldrá del TLCAN, y ha amenazado con imponer aranceles de hasta 35\% a algunas exportaciones clave de México, como automóviles.

En política migratoria, la amenaza de gravar las remesas para financiar el muro en la frontera implicaría un golpe directo a los ingresos de familias de escasos recursos. Además, si Washington implementa una política de deportaciones más agresiva, miles de migrantes regresarían a zonas con mercados de trabajo muy deprimidos.

Así las cosas, las expectativas para el desempeño de la economía en 2017 son poco optimistas. Por un lado, los especialistas del sector privado esperan un repunte de la inflación por encima del objetivo inicial, y un crecimiento del PIB por debajo de $2 \%$. En estas condiciones, resulta casi quijotesco esperar que el gobierno incremente su gasto en CTI.

Por otro lado, el escepticismo de Trump con respecto al conocimiento científico, su animadversión hacia la cooperación internacional en todos los campos y, sobre todo, su desconfianza y hostilidad hacia México y hacia los mexicanos que viven en Estados Unidos también podrían complicar la agenda de cooperación en CTI de dos maneras. Una, la posible reducción de fondos federales para esa cooperación. La otra, grandes restricciones a la entrada de estudiantes, profesores y científicos mexicanos a Estados Unidos, así como la deportación de un número indeterminado de ellos. Por ejemplo, las autoridades ya han deportado a algunos dreamers, es decir, jóvenes indocumentados que llegaron de niños a Estados Unidos y a quienes Barak Obama dio una amnistía para seguir estudiando o trabajando de manera legal.

Finalmente, y por el lado positivo, el sistema institucional estadounidense es sumamente descentralizado, por lo que más allá de lo que decida el gobierno de Trump otros actores relevantes para la cooperación en CTI -academia, empresas, centros de investigación, laboratorios privados- podrían retomar la estafeta que dejara caer el aislacionismo de Washington.

\section{Conclusiones}

Hemos subrayado la urgencia de que México incremente su inversión -tanto pública como privada- en investigación y desarrollo y específicamente en CTI. Una economía emergente y semi industrializada como la mexicana no puede aspirar a insertarse en una senda de desarrollo robusta dejando de lado sus capacidades

13- Por ejemplo, eliminar el Capitulo 19 sobre Revisión y Solución de Controversias en Materia de Cuotas Anti-Dumping y Compensatorias. 
de CTI. El compromiso con este empeño tiene que asumirse de manera nacional con esfuerzos dedicados explícita y fuertemente a ello de manera sostenida. No es algo que pueda obtenerse de manera incidental, por mera voluntad de socios comerciales. Claro que el potencial de la cooperación de los socios norteamericanos en este campo es muy grande, pero hay que diseñar y aplicar una política para ello. Debería ser evidente para las autoridades económicas del país que es indispensable impulsar el sistema nacional de innovación (CTI) si México ha de comenzar a cerrar las brechas de desarrollo con los vecinos del norte.

Desafortunadamente, la aplicación de la política fiscal en México de tiempo atrás no apunta en esa dirección. El gasto público en CTI y, en general, en la formación de capital se recorta en momentos de dificultades presupuestales, y no se restablece del todo después. Como ejemplo desde 2009 el balance primario del sector público ha sido deficitario en un marco de caída de los ingresos fiscales petroleros, de alza del gasto corriente y recorte de la inversión pública. La recomposición del gasto público en contra de la formación de capital fijo afecta la calidad de la infraestructura, y con ello, merma el crecimiento potencial de la economía mexicana. Pero tampoco el gasto del sector privado en CTI en México tiene un comportamiento dinámico. Ello se debe en gran medida a que nuestro sector exportador apostó, y sigue apostando, por una inserción en la economía mundial basada en los bajos salarios de los trabajadores mexicanos como "ventaja" competitiva. Bajo ese modelo el gasto en CTI, tiene escaso interés para nuestras empresas. Por demás grave es que este modelo seguido por México simplemente ha fracasado en su intento de llevar al país a un desarrollo compartido, robusto. Urge cobrar conciencia de que la competitividad mexicana en los mercados mundiales debe basarse en la creatividad, el valor agregado y la calidad. Para lograr esta competitividad, digamos auténtica y no espuria basada en bajos salarios, es indispensable orientar recursos y voluntades de manera intensa, sostenida por el sector público, el sector privado y la sociedad civil para impulsar la innovación, la ciencia y la tecnología para el bienestar de los mexicanos.

En ese sentido, las políticas de desarrollo productivo cobran un papel fundamental, de manera que se dinamice el crecimiento y fortalezcan los eslabonamientos productivos de las industrias en las que la economía mexicana tiene una ventaja comparativa; por ejemplo, (i) manufactura de productos con un coeficiente peso/valor alto (la producción de automóviles, pantallas planas y electrodomésticos de gran tamaño); (ii) producción de empresas que operan con procedimientos just-in-time y cuya producción está sujeta a cambios frecuentes en el diseño (auto-partes); (iii) productos que requieren una sólida participación directiva para cumplir con elevados estándares de calidad (industria aeroespacial e instrumentos médicos), y (iv) productos manufacturados en los que es importante proteger los derechos de propiedad (un análisis más detallado se encuentra en Watkins (2007)).

Es fundamental, también, que la implementación de las políticas de desarrollo productivo se lleven acorde a lo estipulado en el Plan Nacional de Desarrollo y al Plan Nacional de Innovación, donde ya se ha hecho mención de la importancia de fortalecer los encadenamientos productivos hacia atrás y hacia delante; de los sectores exportadores al resto de la actividad productiva. ${ }^{14}$

De manera que, aunque los antecedentes que han marcado un parteaguas para el fomento a y cooperación en el campo de la CTI son relevantes y pueden ser mejorados, es claro que los esfuerzos deben ser redoblados con la finalidad de ampliar el impacto que las políticas implementadas en este rubro sobre el crecimiento y desarrollo a largo plazo, en particular para la economía mexicana. 


\section{Referencias}

Antal, Edit y Fidel Aroche. 2010. La cooperación en ciencia y tecnología en América del Norte y Europa. Ciudad de México: CISAN-UNAM.

Atkinson, Robert. 2014. Understanding the U.S. national innovation system. Washington DC: The Information Technology \& Innovation Foundation.

Colmex. 2014. Informe de labores del programa interinstitucional de estudios sobre la región de América del Norte 1993-2013. Ciudad de México: Colmex.

Cimoli, Mario. 2000. Developing Innovation Systems: Mexico in the global context. Londres: Pinter Series. Dutrénit, Gabriela. 1994. Sistema nacional de innovación. Revista de Comercio Exterior, 666-668.

Dutrénit, Gabriela, Mario Capdevielle, Juan Manuel Corona. 2010. El sistema nacional de innovación mexicano. En El Sistema nacional de innovación mexicano: instituciones, políticas, desempeño y desafíos, compilado por Gabriela Dutrénit, Mario Capdevielle, Juan Manuel Corona. México: Universidad Autónoma Metropolitana, 64-90.

Dutrénit, Gabriela y Marcela Suárez. 2015. Entre flujos de conocimiento, asimetrías y espacios de oportunidad: la CTI en América del Norte. Ciudad de México: Senado de la República LXII Legislatura.

Dussel Peters, Enrique y Kevin Gallagher. 2013. El huésped no invitado del TLCAN: China y la desintegración del comercio en América del Norte. CEPAL, 110: 85-111.

Fernádez-Ribas, Andrea y Philip Shapira. 2009. The role of national and regional innovation programmes in stimulating international cooperation in innovation. International Journal of Technology Management, 48(4): 473-498. DOI: https://doi.org/10.1504/IJTM.2009.026690

Freeman, Christopher. 1987. Technology and economic performance: Lessons from Japan. Londres: Pinter. FCCyT. 2013. Proyecta 100,000. Hacia una región del conocimiento. Propuesta del grupo de consulta mexicano del foro bilateral sobre educación superior, innovación e investigación (FOBESII), FCCyT. http://redtalentos.gob.mx/documentos/reuniones/americas2014/9proyecta_100000_hacia_una_region_del_conocimiento.pdf (02 de noviembre, 2017).

Gurría, Ángel. 2009. Innovación regional en México. Ponencia presentada en Estudio de la OCDE para la Innovación regional en 15 estados mexicanos, México.

Martínez-Noya, Andrea y Esteban García-Canal. 2017. Location, shared suppliers and the innovation performance of R\&D outsourcing agreements, Industry and Innovation, 25(3): 308-332. DOI: https:// doi.org/10.1080/13662716.2017.1329085

Moery, David y Nathan Rosenberg. 1993. The U.S. national innovation system. En National innovation systems. A comparative analysis, coordinado por Richard Nelson. Nueva York: Oxford University Press, 29-75.

Moreno-Brid, Juan Carlos. 2015. Política macro e industrial para un cambio structural y crecimiento: gran pendiente de la economía mexicana. Revista Problemas del Desarrollo. 185(47): 59-80.

Nelson, Richard. 1993. National innovation systems. A comparative analysis. Nueva York: Oxford University Press.

Niosi, Jorge, André Manseau y Benoit Godin. 2000. Canada's national system of innovation. Montreal: McGill-Queen's University Press. 
Lederman, Daniel y William Maloney. 2006. Innovation in Mexico: NAFTA is not enough. En Global integration and technology transfer, compilado por Bernard Hoekman y Beata Smarzynska Javorcik. Washington, D.C.: World Bank and Palgrave Macmillan, 327-331.

Lemarchand, Guillermo. 2010. Science, technology and innovation policies in Latin America and the Caribbean during the past six decades. Science Policy Studies and Documents in LAC. 15-140.

Lundvall, Bengt-Åke. 1992. National innovation systems: Towards a theory of innovation and interactive learning. Londres: Pinter.

OECD. 1997. National innovation systems. París: OCDE Publishing.

OECD. 2009. Regional Innovations in Mexico: 15 Mexican States. Paris: OECD Reviews of Regional Innovation.

OECD. 2017. Economic surveys: México 2017, boosting productivity through integration into Global Value Chains. París: OCDE Publishing.

Oregioni, María y María López. Cooperación Internacional en Ciencia y Tecnología: La voz de los investigadores. Revista Iberoamericana de Ciencia, Tecnología y Sociedad. 8(22): 57-73.

Park, Walter. 2011. Technology trade and NAFTA. Washington, D.C.: Nova Science Publishers.

Sánchez, Isaac y Rosa García. 2015. Geografía del crecimiento económico y del (sub) desarrollo científico, tecnológico y de innovación regional en México. En Desarrollo económico y cambio tecnológico. Teoría, marco global e implicaciones para México, compilado por Arturo Ranfla, Miguel Rivera y René Caballero. México: UNAM y Juan Pablos Editor, 265-302.

Sánchez, Isaac y Juan Carlos Moreno-Brid. 2016. El reto del crecimiento económico en México. Industrias manufactureras y política industrial. Revista Finanzas y Política Económica, 8(2): 271-299. DOI: http://dx.doi.org/10.14718/revfinanzpolitecon.2016.8.2.4

Sebastián, Jesús. 2000. Las redes de cooperación como modelo organizativo y funcional para la I+D. Redes. 7(15): 97-111.

SRE. 1972. Acuerdo de cooperación científica y técnica entre los Estados Unidos Mexicanos y los Estados Unidos de América. http://cooperacion-internacional.conanp.gob.mx/docs/Acuerdo\%20de\%20Cooperación\%20Técnica\%20y\%20Cient\%C3\%ADfica\%20México\%20-\%20EUA.pdf (20 de agosto, 2017). SRE. 2005. Declaración Conjunta. Alianza para la seguridad y la prosperidad de América del Norte. https:// mex-can.sre.gob.mx/images/docs/DECLARACION-CONJUNTA-Cancun-2006.pdf (20 de agosto, 2017). SRE. 2011. Alianza México-Canadá. https://mex-can.sre.gob.mx/index.php/alianza-mexico-canad (20 de agosto, 2017).

SRE. 2011. Acuerdo de cooperación en materia de investigación e innovación entre el Gobierno de Quebec y el Gobierno de los Estados Unidos Mexicanos. https:/embamex.sre.gob.mx/canada/index.php/es/ prensa/comunicados/11491-com039-16 (21 de agosto, 2017).

US Department of State. 2005. Joint statement by president Bush, president Fox, and prime Minister Martin: Security and prosperity partnership of North America, https://2001-2009.state.gov/p/wha/rls/ prsrl/2005/69850.htm (20 de agosto, 2017).

Watkins, Ralph. 2007. El reto de China a las manufacturas de México. En Oportunidades en la relación económica y comercial entre China y México, compilado por Enrique Dussel Peters. México: CEPAL, 137-164. 